\title{
A Novel Architecture of Telecommunication Networks for Next Generation Internet
}

\author{
Fengchen Qian ${ }^{1, *}$, Yalin $\mathrm{Ye}^{1}$, Ning Shan ${ }^{2}$, Bing $\mathrm{Su}^{1}$ \\ ${ }^{1}$ School of Information and Communication, National University of Defense Technology, 710106 Xi'an Shaanxi, China \\ ${ }^{2}$ Armed Police Force Engineering University, 710086 Xi'an Shaanxi, China
}

\begin{abstract}
In this paper, we present a novel DC-centric architecture of telecommunication networks for next generation Internet. Data flow become the major traffic in existing telecommunication networks. Traditional computer networks and telecom networks meet many challenges in high-quality service, innovation, evolution, and management. Based on analysis existing telecom networks' challenges, a DCcentric telecom network architecture with splitting the data plane from the control plane is proposed. The DC-centric telecom network is a widely-distributed data center network (DCN), which is composed of thousands of public or private DCs. Each DC not only plays the role of storage and computing, but also is a network node of data aggregation, switching, and routing. We also design an optical switching, which is evaluated by experiment.
\end{abstract}

\section{Introduction}

With the development of cloud computing, mobile internet, Internet of Things and virtual reality technologies, data flow become the major traffic in existing telecommunication networks. At the same time, driven by a rapid growth of Internet-based applications, data center (DC) has emerged as a crucial infrastructure, and been receiving significant research interest both from academia and industry. Big bang data is leading the many large Information Technology (IT) companies to invest more on the DC [1,2]. DCs are experiencing a rapid growth in both scale and complexity. The forecast by the Cisco Global Cloud Index (GCI) presents that hyperscale data centers will grow from 259 in number at the end of 2015 to 485 by 2020 . The amount of annual global data center traffic in 2020 will be triple $4.7 \mathrm{ZB}$ estimated in 2015 to reach to 15.3 ZB. By 2020, 92 percent of workloads will be processed by cloud data centers in the forecast [3]. Telecommunication networks play a pivotal role in connecting users to DCs, DC to DC, and so on. Despite its importance, the architectures of today's telecommunication networks are still far from being ideal. Many deficiencies, as highly heterogeneous, low reliability, longer latency, low energy efficiency, ossification effect and hard to manage, are becoming obstacles to the development of the next generation Internet.

\section{Architecture and challenges of existing telecommunication networks}

Traditional telecommunication networks have been built using a very common design that involves the implementation of a three-tier architecture. Figure 1 shows a conventional telecommunication network topology. In this architecture, the network consists of three layers in the horizontal direction, called as public transport layer, service bearer layer, and users layer respectively.

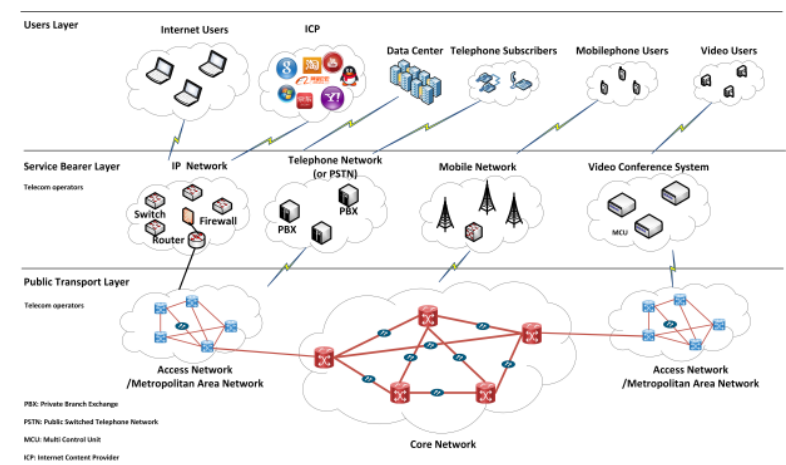

Fig. 1. Architecture of Existing Telecommunication Networks.

Public transport layer is the base of telecommunication networks and provides the transmission channel for all kinds of telecommunication service networks, such as voice, data, image, or video networks, and so on. It is usually built by the operators or governments for all service providers. Nowadays, the public transport layer widely utilizes high-speed optical communication technologies, as Synchronous Digital Hierarchy (SDH), Wavelength Division Multiplexing (WDM), Automatically Switched Optical Network (ASON), Optical Transport Network (OTN), etc[4,5].

Service bearer layer provides all kinds of services such as data communication, voice communication, video communication, and so on. Based on Internet

* Corresponding author: fchen_qian@163.com 
Protocol (IP) computer networks, traditional telephone networks, mobile networks and special video conference systems are interconnected to a completely and heterogeneous service bearer layer.

Users layer consists of consumer users and business users. The consumer users are the end-users, as Internet users, telephone, mobile phones, or video users. The business users are enterprise users and Internet Content Providers (ICP), for example, Baidu, Google, or Tencent. They all are connected to the networks built by telecom operators.

This telecommunication network built by operators consists of multi-level networks, public transport network is at bottom. Service bearer networks as IP network, or mobile network, are at middle level. However, some problems posed by this architecture are the limitation to evolution of the communication infrastructure.

\subsection{Unable to meet the requirements of high- quality service}

To many new technologies and applications such as 5G, unmanned aerial vehicle (UAV), and cloud computing in the Networked Society, designers of telecom are engaged in cope with the needed dimension of latency with sub millisecond(ms) and improving fault tolerance. The current architecture is too complex and includes a large amount of gateway devices so that it is almost impossible to achieve those goals.

\subsection{Causing the network ossification effect}

Most of telecom devices using vertical integration, mean that the control plane and the data plane are bundled inside those devices, reducing flexibility and hindering innovation and evolution of the networking infrastructure. For example, in current IP networks, deploying a new routing algorithm maybe take 5 to 10 years. A complete changing the wide Internet IP architecture is regarded as a daunting task [6,7].

\subsection{Increasing capital and operational cost}

Many control algorithms and communicational protocols are integrated into hardware components inside devices, such as Application Specific Integrated Circuits (ASICs). For example, more complex processing must be implemented by expensive devices. Moreover, changing configuration needs hugely labor intensive adjustments [8].

\subsection{Hard to manage}

For example, traditional IP networks' complexities in configuration. To express the desired high-level network policies, network operators need to configure each individual network device separately using low-level and often vendor-specific commands. Most of telecom networks would be statically configurated to ensure the network's stability. Automatic reconfiguration and response mechanisms are virtually non-existent in current networks. Enforcing the required policies in such a dynamic environment is therefore highly challenging [6].

\section{A novel DC-centric architecture of telecommunication networks}

\subsection{DC-centric Architecture}

With the business and application driving scalability, agility, cost-savings, energy efficiency and differentiation services needs for telecom networks, more requirements are emerging $[9,10,11]$, which can be summarized to the following as simplification, virtualization, programmability, automation, and opensource. In consideration of next generation Internet's requirements and cost and configurability, different service bearer networks and transport network would be merged into a public communication network, which can carry all kinds of services and meet those strict requirements from new applications, such as $5 \mathrm{G}$, UAV, etc.

Taking into account the trend that data flow is the major traffic for telecom networks, we present a novel architecture based on DC to reduce heterogeneity and complexity, as shown in Figure 2.

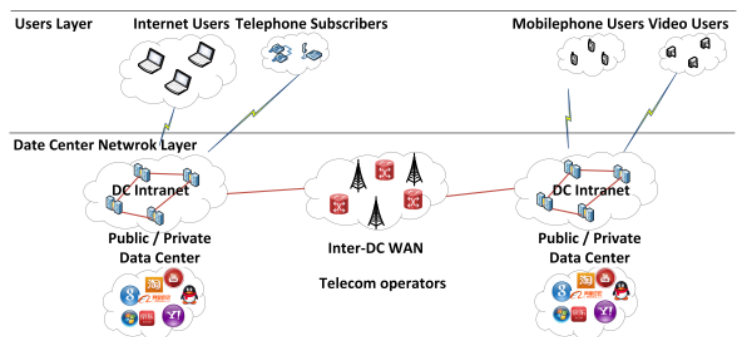

Figure. 2. A Novel DC-centric Architecture of Telecommunication Networks.

The DC-centric telecom network consists of two layers, which are data center network layer and users layer respectively.

\subsection{Physical DCN Layer}

DCN layer is a public transport channel for users' data, which is composed of thousands of public or private DCs. This structure is a widely-distributed data center network (DCN), including intra-DCN and Inter-DCN. Each DC not only plays the role of storage and computing, but also is a network node of data aggregation, switching, and routing. Large scale DCs will act as core nodes, and small DCs like enterprise private DCs or personal DCs will act as access nodes. All users are connected to local DCs, and DCs can be interconnected inter-DC wide area network (WAN). DCs will be built by ICP, telecom operators, government, and even individuals.

Intra-DCN is a local area computer network which connects thousands, even hundreds of thousands of computational, storage, and switching nodes with copper cables or optical fibers $[1,2]$. Intra-DCN architecture is 
an important component of large-scale data centers and has a great impact on the general data center performance and throughput [12]. Traditional data center networks have been built using a very common design that involves the implementation of a two-tier or threetier architectures. Figure 3 shows a conventional data center network topology [13].

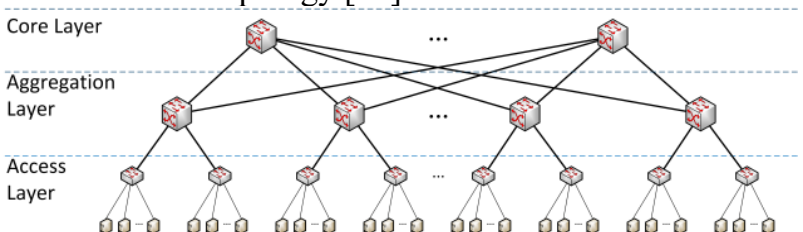

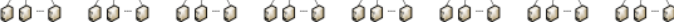

Figure. 3. A conventional data center network topology.

In what follow, many modern DCN architectures are proposed to meet the requirements of high-quality services, and have full bandwidth, good scalability, high utilization, easy cabling, and low cost. Figure 4 shows the classification according to the different types of the routing protocols of the most recent data centers[14,19].

Inter-DCN will be used to connect DC to DC, which is achieved with existing optical network infrastructure. Because optical fiber can provide huge bandwidth and ultra-long transmission distance, it can be deployed to interconnect DCs to meet the requirements of traffic surge and balancing [15]. Due to super-high switching capacity and flexible multiplexing capability, all-optical switching technologies provide the possibility for large scale inter-DCN. Optical circuit switching (OCS) and optical packet switching (OPS) are considered as the most appropriate switching technologies for inter-DCN.

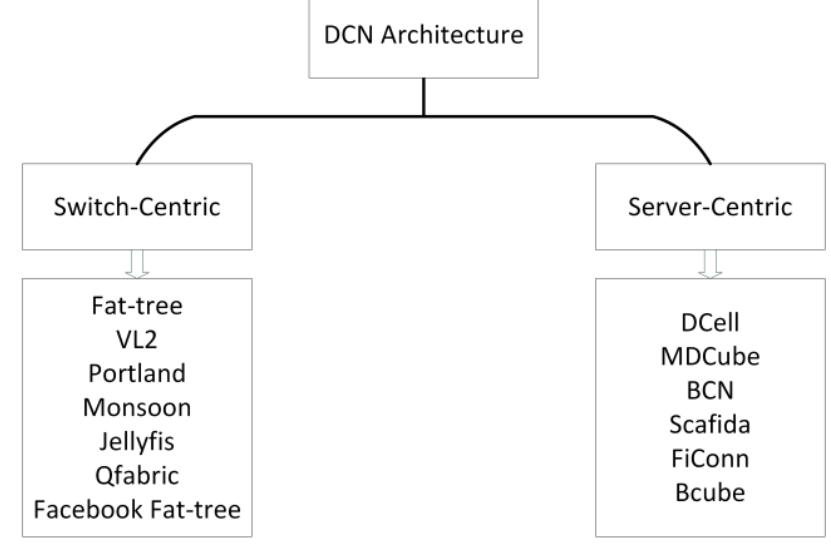

Figure. 4. The classification of DCN architectures

\subsection{Network Control and Management}

In DC-centric telecom network, inter-DCN and intraDCN should be jointly considered. Traditional telecom networks have become increasingly difficult to evolve in its physical infrastructures and protocols. The idea of "programmable networks", called Software-Defined Networking (SDN), has been proposed as a solution to facilitate network evolution[8,16]. Compared to conventional network, SDN has a better prospect for DC-centric telecom network. The fundamental principle of SDN is that splitting the data plane from the control plane in networks to implement the data handling rules as software modules rather than embedding them in hardware, shown as Figure 5.

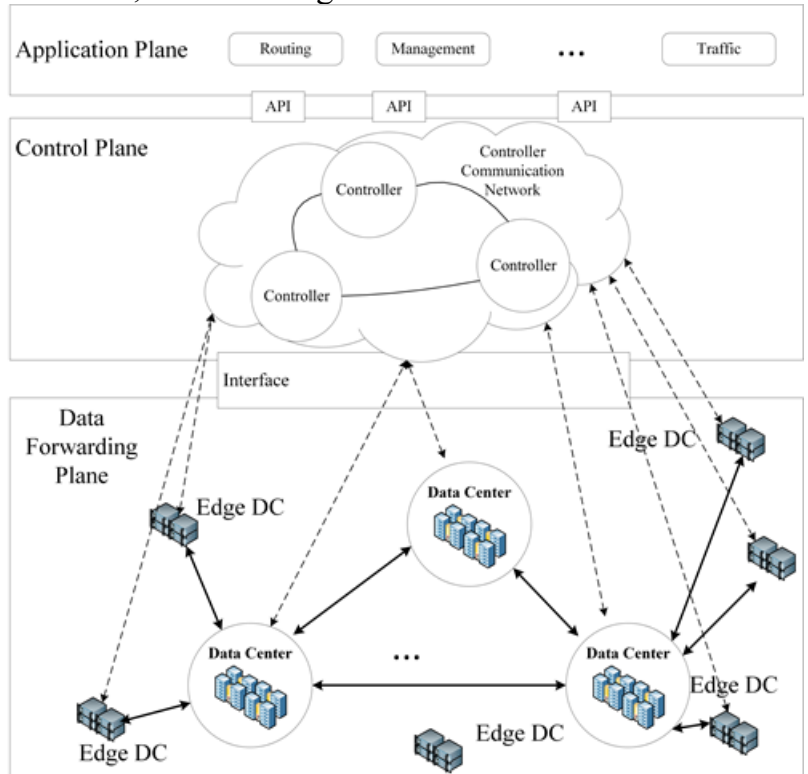

Figure. 5. View of an SDN architecture.

A separate control plane can implement routing, system configuration, traffic management, and other control and management functions, that enables innovation and evolution of networks. The control plane provides a view of the whole network, that is helpful to automatic provision without a device-centric configuration on each entity[8].

\subsection{Advantage of DC-centric Telecom Network}

The architecture of DC-centric telecom network has some advantages includes:

(1) From management perspective, DC-centric telecom network needs dramatically simplify network management and enables innovation and evolution. Because of control plane's separation, DC-centric telecom network enables operators to have more efficient and instant access to the configuration modifications, that introduces flexibility, scalability, manageability and security for DC-centric telecom network operators [17].

(2) From cost and energy perspective, DC-centric telecom network enables operators purchase hardware devices separately from control software and allows operators to easily deploy different vendors' devices instead of the same vendor's high-performance switchers/routers. hardware white-box and software modularity and reusability, will reduce capital and operational cost. The power optimization for data plane and control plane can are jointly considered. The energy consumption models built in control plane related to multi-path routing, traffic management, and hardware resources control would be used to optimize the energy efficiency [18].

(3) From user and tenant perspective, DC-centric telecom network hides the low-level physical details to high-level applications. DC-centric telecom network can rely on network resource virtualization and visualization to well support users' applications and cross-tenant data 
center optimization. DC-centric telecom network allows software developers or DC user to utilize network resources in the same easy manner as they do on storage and computing resources[16].

\subsection{Design on Key Components}

Optical switching is the key technology for OCS/OPS. We deploy an all-optical switching with tunable wavelength convertor (TWC) which can avoid blocking and realize capability of broadcast, shown as Figure 6 .

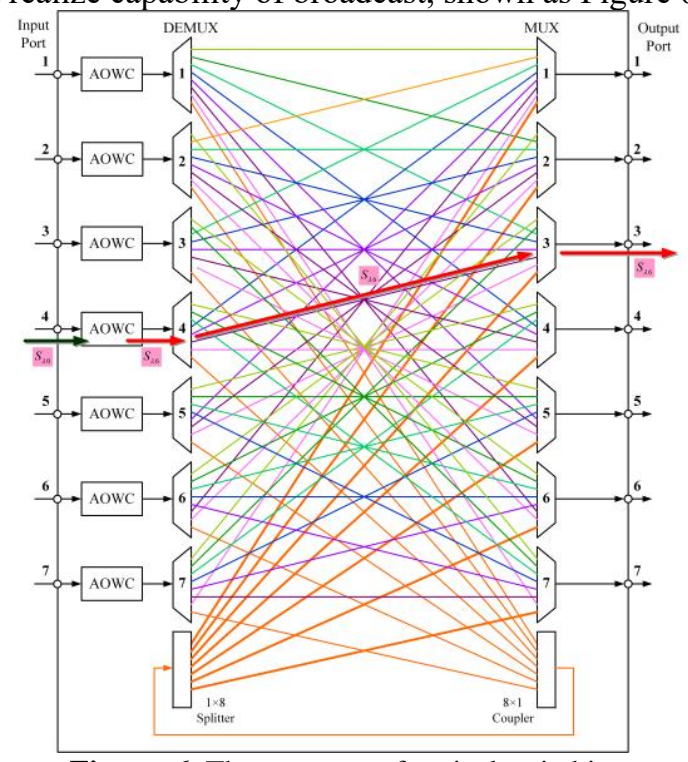

Figure. 6. The structure of optical switching

This is one of optical switching structures with wavelength convertor and arrayed-waveguide grating (AWG). TWC can be instead of Fast Tunable Laser module or Wavelength Selective Switching (WSS) which can implement fast wavelength conversion in less than $10 \mathrm{~ms}$. So the total switching time can be declined to $10 \mathrm{~ms}$. The optical signal $\mathrm{Q}$ factor of every output ports after TWC and AWG are shown as Figure 7.

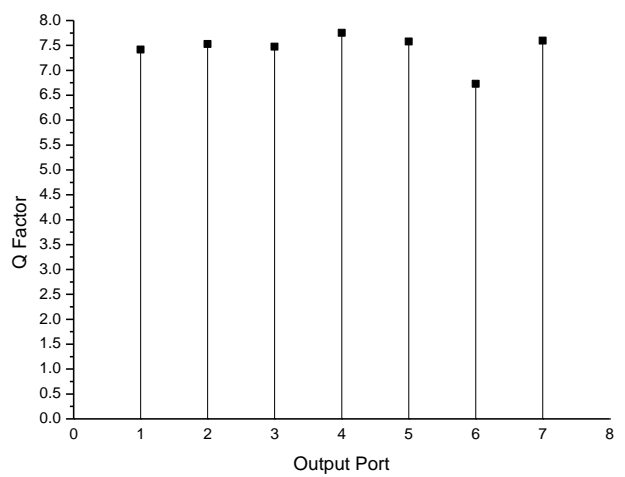

Figure. 7. the optical signal Q factor of every output ports The difference of $Q$ factor of each port output signal is less than $1 \mathrm{~dB}$, and the performance of each channel is uniform.

\section{Conclusion}

We propose a novel DC-centric telecom network architecture with splitting the data plane from the control plane. We summarize the architecture of existing telecom networks and analyze those challenges. The DCcentric telecom network consists of two layers, which are data center network layer and users layer respectively. DCN layer is composed of thousands of public or private DCs and is a widely-distributed data center network (DCN), including intra-DCN and Inter-DCN. Each DC not only plays the role of storage and computing, but also is a network node of data aggregation, switching, and routing. There are some advantages of the architecture of DC-centric telecom network, such as simplifying network management, optimizing the energy efficiency, supporting rapid innovation, and so on.

\section{References}

1. Sankaran, Ganesh C., and K. M. Sivalingam, Photonic Netw. Commun. 33, 2(2017).

2. Xia, Wenfeng, et al., IEEE Commun. Surveys Tuts. , (2017).

3. Cisco, Cisco Global Cloud Index: Forecast and Methodology, 2014-2019. White Paper, (2014).

4. Winzer, Peter J., IEEE Commun. Mag. 48, 7(2010).

5. Sexton, M. J., \& Reid, A. B., Transmission networking: SONET and the synchronous digital hierarchy(Artech House, Inc., 1992).

6. Kreutz D., Ramos F. M., Verissimo P. E., Rothenberg C. E., Azodolmolky S., Proceedings of the IEEE 103, 1 (2015).

7. Barrett R., Haar S., Whitestone R., Interactive Week, 25 (1997).

8. Hu F., Hao Q., Bao K., IEEE Commun. Surveys Tuts., 16 (2014).

9. 2017 Next Gen Data Center Networking Report Online

Edition, https://www.sdxcentral.com/reports/2017/next-gendata-center-networking.

10. Hammadi, A., \& Mhamdi, L., Comput. Commun., 40 (2014).

11. 3 Trends Guide Development of Next-Gen DC Networks, http://eblog.huawei.com/3-trends-guidedevelopment-next-gen-dc-networks.

12. Chen K., Singla A., Singh A., Ramachandran K., Xu L., Zhang Y., Chen Y., IEEE/ACM Trans. Networking 22, 2 (2014).

13. Al-Fares M., Loukissas A., Vahdat A., ACM SIGCOMM Comp. Com. 38, 4 (2008).

14. Bilal K., Khan S. U., Kolodziej J., Zhang L., Hayat K., Madani S. A., Chen D., ECMS, (2012).

15. Luijten R., Denzel W., LEOS, 1 (2014).

16. Nunes B. A. A., Mendonca M., Nguyen X. N., Obraczka K., Turletti T., IEEE Commun. Surveys Tuts. 16, 3 (2014).

17. Bari M. F., Boutaba R., Esteves R., Granville L. Z., Podlesny M., Rabbani M. G., Zhani M. F., IEEE Commun. Surveys Tuts. 15, 2 (2013). 
18. Xie K. U. N., Huang X., Hao S., Member S, IEEE Access, 4 (2016).

19. Alshahrani R. A., Delay Modeling in Data Center Networks: A Taxonomy and Performance Analysis , Kent State University, 7-8(2013) 